\title{
In Situ Measurement of the Toughness of the Interface Between a Thermal Barrier Coating and a Ni Alloy
}

\author{
Christoph Eberl, ${ }^{\dagger, \ddagger \S}$ Xi Wang, ${ }^{\ddagger, \uparrow}$ Daniel S. Gianola, ${ }^{\ddagger, \|}$ Thao D. Nguyen, ${ }^{\ddagger}$ Ming Y. He, ${ }^{\dagger \dagger}$ Anthony G. Evans, ${ }^{\dagger \dagger}$ \\ and Kevin J. Hemker ${ }^{\ddagger}$ \\ ${ }^{\ddagger}$ Department of Mechanical Engineering, Johns Hopkins University, Baltimore, Maryland 21218-2682 \\ ${ }^{\S}$ Institute for Applied Materials, Karlsruhe Institute of Technology, Karlsruhe 76131, Germany \\ "Institute of Mechanics, Chinese Academy of Science, Beijing 100190, China
}

"Department of Materials Science \& Engineering, University of Pennsylvania, Philadelphia 19104-6272, Pennsylvania

"Department of Materials, UCSB, Santa Barbara 93106-5050, California

\begin{abstract}
A flexural test for the in situ measurement of the delamination toughness of the interface between a thermally grown oxide and a bond coat in the presence of a thermal barrier coating (TBC) has been implemented. To accomplish the testing, a section of the substrate was removed by microelectro-discharge machining and a precrack introduced through the TBC by center point loading. This was followed by application of an asymmetric single-point load to extend a delamination along the interface. A displacement and strain mapping method was used to locate the delamination and to ascertain its extension. To relate the energy release rate and mode mixity to the crack extension and the loads, a finite-element method was implemented. The ensuing fracture resistance was found to vary along the interface with values in the range of $25-95 \mathrm{~J} / \mathrm{m}^{2}$.
\end{abstract}

\section{Introduction}

$\mathrm{T}$ HE integrity of metal/oxide interfaces governs the viability of many multilayer systems and governs the lifetime of such applications. The mechanics dictating the energy release rates and mode mixities have been comprehensively established for many salient problems. ${ }^{1}$ However, implementation has often been limited by the difficulty in measurement of the interface fracture toughness at the relevant mode mixity. There are literally hundreds of different methods for characterizing adhesion, and a recent review of interfacial toughness measurements for thin films on substrates has identified six different categories of tests for measuring critical values of stress intensity or energy release rates. ${ }^{2}$ These categores include: superlayer, indentation, combined superlayer indentation, scratch, bulge and blister, and sandwich specimen tests. Each test comes with inherent advantages and disadvantages and no one methodology has come to be known as the gold standard for measuring thin film or coating adhesion. For the thermal barrier coatings (TBCs) considered in the current study, techniques involving notch flexure sandwich specimens are most salient.

Variants on a notch flexure test have been widely exploited, particularly for interfaces in multilayer semiconductor devices. $^{3,4}$ This test typically measures the mixed mode toughness, at a phase angle of approximately $\Psi \approx 42^{\circ}-50^{\circ}$. The method is

T. Pollock - contributing editor

\footnotetext{
Manuscript No. 28992. Received December 2, 2010; approved March 26, 2011.

This work was supported by AFOSR under the MEANS-2 Program (Grant No. FA9550-05-1-0173) and by ONR (Grant No. N00014-08-1-0454).
}

Author to whom correspondence should be addressed. e-mail: chris.eberl@ kit.edu applicable to interfaces within planar devices, primarily on thin substrates. It cannot be applied to either thick or curved substrates. This paper presents a new flexure test method that obviates this limitation and allows in situ measurement on films and coatings attached to substrates; the mechanics-based design for which is described in a companion article. ${ }^{5}$ In the current study, this method has been used to measure the toughness between an oxide coating and a relatively small diameter cylindrical substrate, the configuration for which is depicted in Fig. 1.

The test design is guided by beam theory solutions for a planar four-point bending configuration. ${ }^{5}$ A three-point bending configuration with asymmetric loading was used in this study but is difficult to solve analytically. For this reason, the simpler case, namely the four-point bending configurations, will be used to explain the experiment. In the absence of residual stress, the energy release rate, $G_{\mathrm{P}}$, for this configuration can be expressed in the normalized form:

$$
G_{\mathrm{P}} E_{2} h_{2}^{3} /(P L)^{2} \equiv \prod\left(\frac{a}{L}, \frac{h_{1}}{h_{2}}, \frac{E_{1}}{E_{2}}, b / L\right)
$$

where $P$ is the load/width, $a$ is the delamination length, $L$ is the span, $b$ is the separation of the inner load points in the fourpoint bending setup, and $h_{1}, h_{2}$ are the thickness of the coating and substrate, respectively, and $E_{1}, E_{2}$ the corresponding values of the Youngzs modulus. The functional form for $\Pi$ has been elaborated elsewhere. ${ }^{5}$ One desirable feature of this test is the fact that the energy release rate diminishes with increase in crack length, so that the delamination progresses only upon increasing the load (stable behavior). In the presence of residual stress, $\sigma_{\mathrm{R}}$, another nondimensional group influences $G_{\mathrm{P}}$, given by: $\Re \equiv \sigma_{\mathrm{R}} h_{1}^{2} / P L$. The influence of residual stress is often overlooked but can be substantial. One demonstrated advantage of the proposed test methodology is that fabrication of the specimen relaxes the residual stresses in the bilayer and greatly simplifies analysis of the experimental data.

In the aero-turbine industry, cylindrical test configurations are routinely used to examine the thermo-mechanical integrity of coated components upon thermal cycling. Such assessment methods are referred to as burner-rig tests. Developing an interface toughness test that can be performed on such configurations as a function of exposure to thermal cycling is of great interest. Extension of this test protocol to commercial engine hardware such as blades and vanes is straightforward. In this study, specific measurements have been performed on a columnar TBC generated by electron beam physical vapor deposition (EB-PVD) on a single crystal, Ni-based superalloy substrate with a NiCoCrAlY bond coat. $^{6}$ During deposition of the 


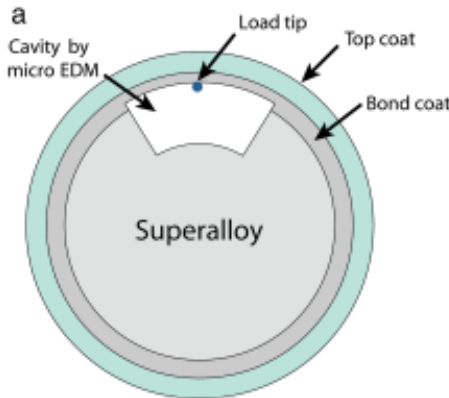

b
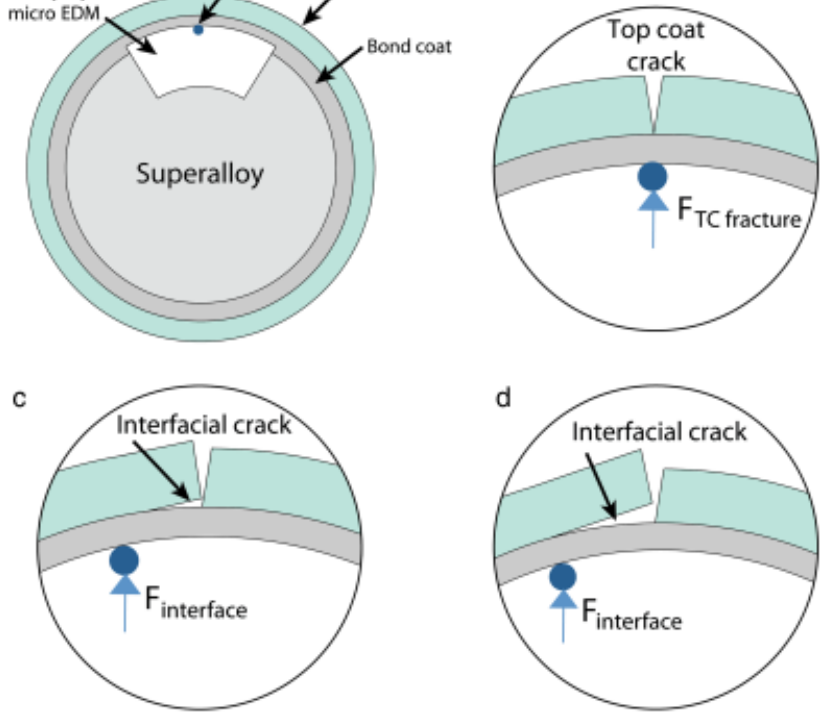

Fig. 1. A schematic of the specimen design and test procedure (a) for the example of a coating on the periphery of a circular substrate. The precracking method is illustrated on the top right (b) and the subsequent asymmetric loading for ascertaining the delamination toughness is on the lower left (c) and right (d).

coating, a thin thermally grown oxide (TGO) layer develops, consisting largely of $\alpha-\mathrm{Al}_{2} \mathrm{O}_{3}$.

To create the test configuration (Fig. 1), a section of the substrate is removed by microelectro-discharge machining ( $\mu$-EDM) to leave an intact bilayer beam along an arc of the surface. The beam segment consists of the coating on the outside and the bond coat on the inside. The specimen is placed within a microtensile test system with the loads applied as indicated on Fig. 1.

The article is organized as follows. The protocol used for the measurements is described. The interface crack extension measurements are presented. Finite-element (FE) results for the energy release rates and mode mixity are summarized and used to ascertain the interface toughness. The results are interpreted in terms of the mechanisms of interface crack extension.

\section{Experimental Protocol}

The specimens, provided by Pratt \& Whitney, included a TBC on the surface that consisted of a $110 \mu \mathrm{m} \mathrm{7 \% -yttrium-stabilized}$ zirconia (7-YSZ) coating, deposited by a EB-PVD process onto a low-pressure plasma-sprayed NiCoCrAlY bond coat. The substrate was a Ni-base superalloy substrate (PWA 1484). Cross-sections were cut and polished from the provided cylindrical burner rig bars and subsequently $\mu$-EDM was used to carve out the substrate and parts of the bond coat underneath the TBC coating (Fig. 1). The final test geometry (Fig. 2(a)) consisted of a doubly end-supported beam of roughly $1 \mathrm{~mm}$ length. A bond coat layer of 35-65 $\mu \mathrm{m}$ (depending on the specimen) was left to support the $110-\mu \mathrm{m}$-thick TBC, resulting in a bilayer beam. The thickness of the cross-section was roughly $550 \mu \mathrm{m}$. Accordingly, the specimens (Fig. 2) have the relative span to thickness ratio, $L / h=6.7$ (Beam 2, Table I) and 7.4 (beam 1 , Table I), coating to substrate thickness ratio, $h_{1} / h_{2} \approx 3$, and modulus ratio, $E_{2} / E_{1} \approx 8$.

The experiment is carried out on a custom-built microtensile setup consisting of a horizontal load train supported by an air bearing. ${ }^{7}$ This configuration provides the alignment of the sample while minimizing friction on the $5 \mathrm{lb}$ load cell. Steel microtips are connected to a piezoelectric stepper motor fixed to a fiveaxis stage to enable the precise alignment of the loading axis. Images are captured during testing by a high-resolution camera attached to an optical microscope. To reduce the influence of vibrations the setup is mounted to an air table. More detailed information on the experimental configuration in provided in Eberl et al. ${ }^{7}$

The test is conducted in two steps. Initially, single-center-point loading is used to induce a vertical crack that extends through the ceramic top coat to the interface (Fig. 1(b)). A single-point load provides a focused stress field with maximum tensile stress at the surface of the TBC. Therefore the TBC is forced to crack at the selected region. ${ }^{8}$ The loading position was chosen to be the center of the beam for all precracking experiments.

Thereafter, an asymmetric single-point load is used to propagate the crack stably along the interface (Figs. 1(c) and (d)). The loading tip is positioned beyond the leading edge of the interface crack away from the center TBC crack during all experiments. This configuration forces the interface crack to extend in the direction of the loading site. The loads at which this happens provide a measure of the toughness as the crack only extends if the load exceeds a critical value.

The key experimental feature needed to analyze the measurements with high fidelity is the ability to use a digital image correlation and tracking (DICT) technique to observe in situ the local displacements. For this purpose, the sample deformation is monitored by a high-resolution CMOS camera (PL-782A, Pixelink, Ottawa, ON, Canada) mounted to an optical microscope (Nikon, Tokyo, Japan) with a field of view ranging from $3.2 \mathrm{~mm} \times 2.3$ $\mathrm{mm}$ to $400 \mu \mathrm{m} \times 290 \mu \mathrm{m}$. The displacement field is calculated by a digital image correlation technique from a series of images, such as those shown in Figs. 2(b) and (c). The achievable displacement resolution is limited by the vibrations, the quality of the optical system, the camera resolution and the signal to noise ratio of each pixel in the image. The experimental configuration used here results in a displacement noise floor with a root mean squared value of roughly $30 \mathrm{~nm}$.

A spatially dense displacement field is calculated from the acquired image sequence in a post process. This is achieved using a virtual mesh of markers with a pitch size of $1-2 \mu \mathrm{m}$, corresponding to roughly 10-20 pixels at the highest magnification, which are defined and tracked by the DICT functions. An example of a mesh used for these measurements is shown in Fig. 2(b). The size of the tracked markers is between $20 \times 20$ and $40 \times 40$ pixels $^{2}$, which is equal to $2.5 \mu \mathrm{m} \times 2.5 \mu \mathrm{m}$ and $5 \mu \mathrm{m} \times$ $5 \mu \mathrm{m}$ at the highest optical resolution used in these experiments. The local displacement gradient is used to calculate the axial strain along the length of the beam, $\varepsilon=\partial u / \partial x$ and the differential displacement along the interface is used to acquire the opening displacement along the crack. The strain and displacement resolutions are increased by averaging the displacement gradient over a finite number of tracked markers and by taking multiple images per load step.

Crack propagation is tracked by analyzing the displacement field using custom Matlab ${ }^{B}$ functions, and the relevant quantities needed for data analysis are defined in Fig. 2(c). One advantage of the DICT method is the output of full-field displacements, providing local information about deformation, as shown in Fig. 3. These computed displacement fields show the precise location of cracks and their evolution. After defining the interface in the acquired images, the crack opening displacement along the interface is measured and correlated to the applied load.

\section{Experimental Results}

As described in previous sections, a precrack is induced close to the geometric center by single-point symmetric loading. This crack follows the columnar microstructure and proceeds from the surface down to the interface. Two different methodologies have been used to extend the interface crack: designated asymmetric single-point loading and symmetric two-point loading. The latter is motivated by the analysis detailed in $\mathrm{He}$ et al. ${ }^{5}$ and the tests conducted in this manner are the most straightforward to interpret. However, asymmetric loading was found to provide much broader experimental flexibility, albeit that the 

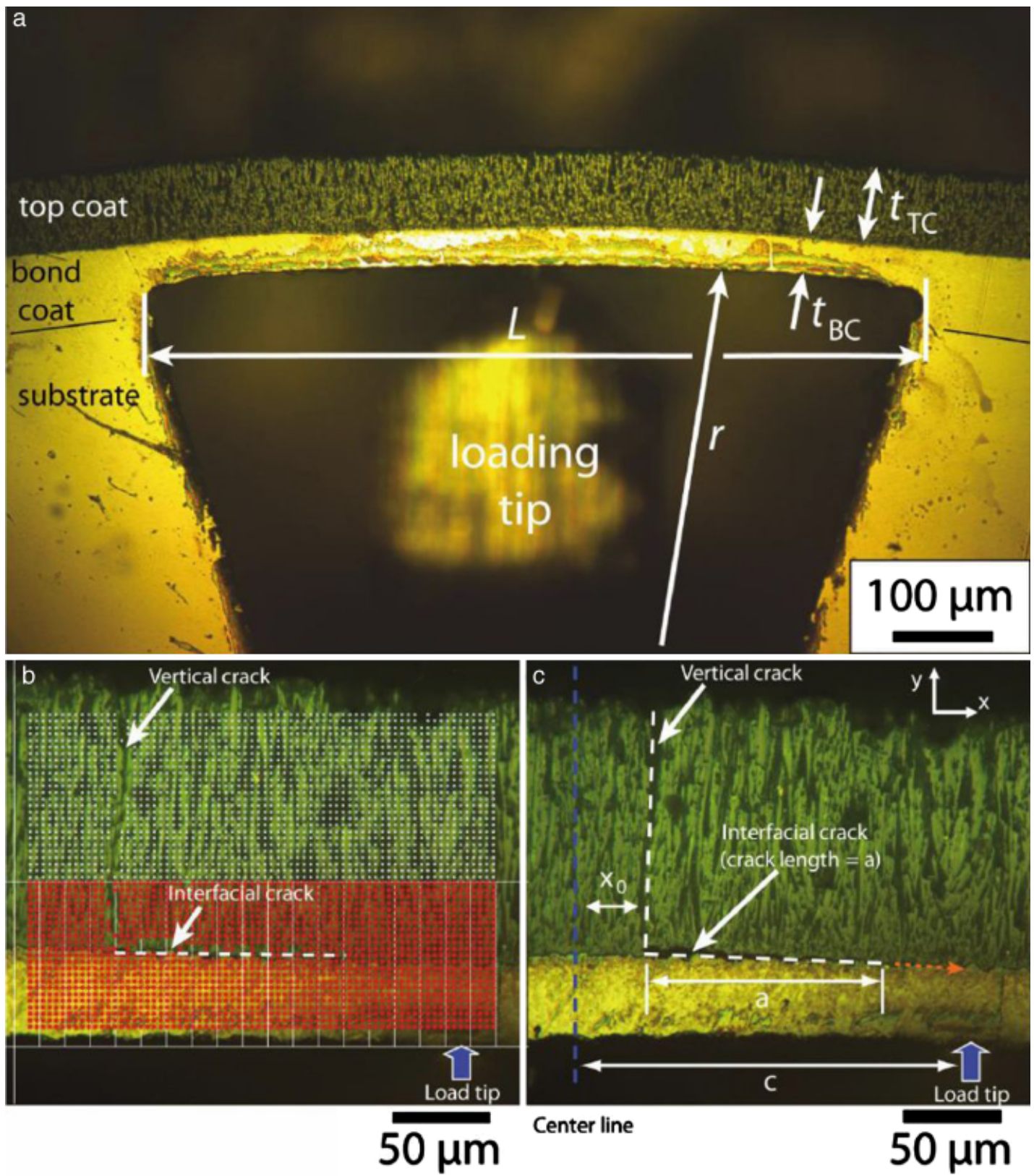

Fig. 2. The optical image on the top (a) is showing the actual configuration used in the measurements comprising a columnar TBC deposited onto a Ni-based alloy and the cut out section below the bond coat. The grid used for the digital image correlation is shown in the lower left (b) where a vertical and interfacial crack has been formed. The geometry is shown in the lower right (c).

interpretation then requires FE solution. In the end, asymmetric loading emerged as the method of choice for the current study.

In this method, a dominant interface crack was generated from the precrack by relocating the load point off-center. This mode of loading caused an interface crack to form and propagate toward the load point in a stable manner. The ensuing interface crack path is shown in SEM images of the cracks in Fig. 4(a). It penetrates the TGO and then follows the interface with the bond coat. The tip region comprises a series of en echelon microcracks traversing the TGO (Figs. 4(b) and (c)), indicative of a mode II mechanism, as elaborated later. To prevent the load/unload/load sequence from influencing the interface at the crack tip, all subsequent tests were conducted in consecutive load steps without unloading in between.

The experimental crack propagation events for two different beams are summarized in Table I. The results from these series of critical events are the basis for the FE-based fracture toughness calculation described in the subsequent section. For beam 1, the aforementioned precrack through the top coat formed at a distance of $x_{0}=29 \mu \mathrm{m}$ away from the geometric center of the beam. The crack proceeded from the TBC surface at the critical load and arrested at the interface between top and bond coat. In the next step the loading tip was offset to $c=36 \mu \mathrm{m}$ from the center of the beam and then loaded again. The loading was conducted in steps to $F=4.9 \mathrm{~N}$ and after unloading the analysis showed that an interfacial crack had nucleated at a load of $F_{\mathrm{c}}=3.1 \mathrm{~N}$. During this event (not listed in Table I), the interface crack had propagated to a length of $a=29 \mu \mathrm{m}$ and extended beyond the position of the loading tip. This was the only time this occurred in all experiments. After nucleating the interface crack, the load tip was again offset to a new location ahead of the interface crack tip at a position $c=78 \mu \mathrm{m}$. After loading to a maximum force of $F=3.7 \mathrm{~N}$, the analysis revealed that the crack had extended after applying a critical force of $F_{\mathrm{c}}=3.3 \mathrm{~N}$ to a length of $a=50 \mu \mathrm{m}$ (not listed in Table I). Continuing the experiment, the tip was then offset to $c=171 \mu \mathrm{m}$ relative to the beam center. Increasing the load in a stepwise fashion led to several consecutive crack propagation events for this configuration. As documented in Table I, the crack propagated by amounts of $a=16,18,16,14$, and $3 \mu \mathrm{m}$ when the critical loads 
Table I. Event table

\begin{tabular}{|c|c|c|c|c|c|c|c|c|}
\hline $\begin{array}{l}\text { Crack } \\
\text { event \# }\end{array}$ & Schematic & $\begin{array}{c}x_{\mathrm{o}} \\
(\mu \mathrm{m})\end{array}$ & $\begin{array}{c}a \\
(\mu \mathrm{m})\end{array}$ & $\begin{array}{c}c \\
(\mu \mathrm{m})\end{array}$ & $\begin{array}{c}\text { Critical load, } \\
P_{\mathrm{c}}(N)\end{array}$ & $\begin{array}{c}\text { Propagation, } \\
\Delta a(\mu \mathrm{m})\end{array}$ & $\begin{array}{c}\text { Interfacial } \\
\text { toughness, } \\
\Gamma\left(\mathrm{J} / \mathrm{m}^{2}\right)\end{array}$ & $\begin{array}{c}\text { Phase } \\
\text { angle, } \psi\left({ }^{\circ}\right)\end{array}$ \\
\hline
\end{tabular}

Beam 1: $L=1.1 \mathrm{~mm} ; \mathrm{W}=550 \mu \mathrm{m} ; h_{b c}=36 \mu \mathrm{m} ; h_{t b c}=112 \mu \mathrm{m} ; E_{b c}=155 \mathrm{GPa}, E \|_{t b c}=20 \mathrm{GPa} ; E_{t b c}^{\perp}=180 \mathrm{GPa}$

1

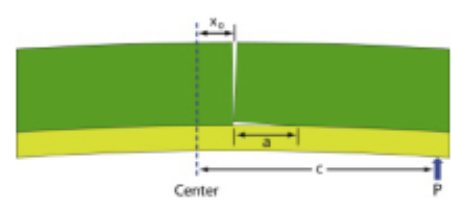

2

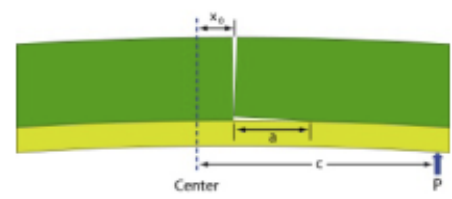

3

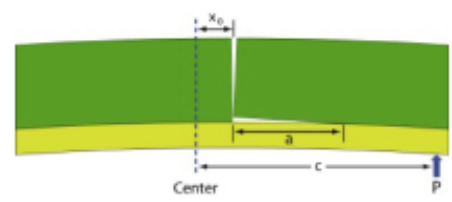

4

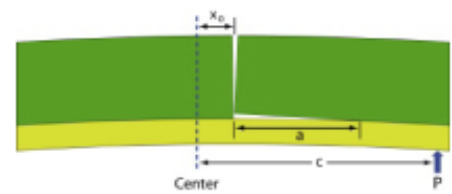

5

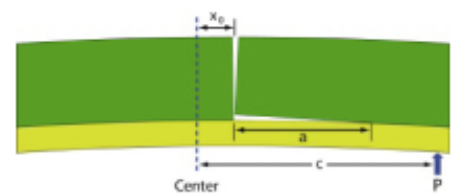

29

60

171

2.3

16

27.1

29

76

171

3.0

18

42.5

18.0

29

94

171

3.5

16

53.6

18.1

29

110

171

4.1

14

67.8

29

124

171

5.1

3

95.2

20.5

Beam 2: $L=1.1 \mathrm{~mm} ; \mathrm{W}=550 \mu \mathrm{m} ; h_{b c}=51 \mu \mathrm{m} ; h_{t b c}=112 \mu \mathrm{m} ; E_{b c}=155 \mathrm{GPa}, E \|_{t b c}=20 \mathrm{GPa} ; E_{t b c}^{\perp}=180 \mathrm{GPa}$

1

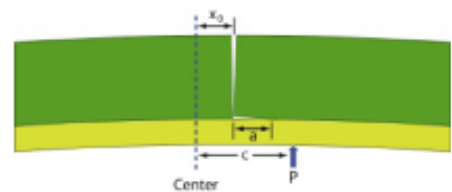

2

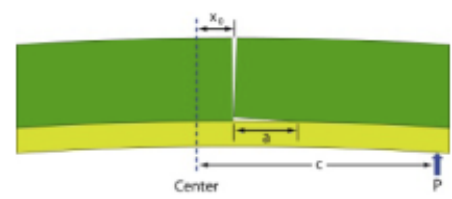

0

450

50

3.2

6

51.1

18.9

0

20

50

3.5

7

63.8

19.1 of $F_{\mathrm{c}}=2.3,3,3.5,4.1$, and $5.1 \mathrm{~N}$ were reached (crack events $1-5$, beam 1).

The second sample (beam 2) was treated in a similar fashion and the crack propagation and critical loads and corresponding fracture energy and phase angle values as calculated by FEA can be found in Table I. First, the beam was center loaded till a crack was induced in the top coat at the very center of the beam with $x_{0}=0 \mu \mathrm{m}$. In a second step, the load was applied at an off center position $c=50 \mu \mathrm{m}$ and the load was increased. As a result, the crack channeled to the interface and extended and to a length of $a=14$ and $20 \mu \mathrm{m}$ at corresponding critical loads of $F_{\mathrm{c}}=3.2$ and $3.5 \mathrm{~N}$ (crack events, beam 2).

\section{Energy Release Rates and Interface Toughness}

The mechanical response of the bilayer beam has been analyzed by the FE method using the commercial code ABAQUS Standard. Only the bilayer beam and part of the support material was simulated in the analysis and the boundary conditions were assumed as rollers. No energy exchanges are allowed between the modeled specimen and the rest of the ring. The finite-element model and the mesh are depicted in Fig. 5. The eight-node biquadratic plane strain elements with reduced integration were used. At the crack tip focused elements were used and the midsize node parameter is chosen to be 0.25 . In these simulations the 

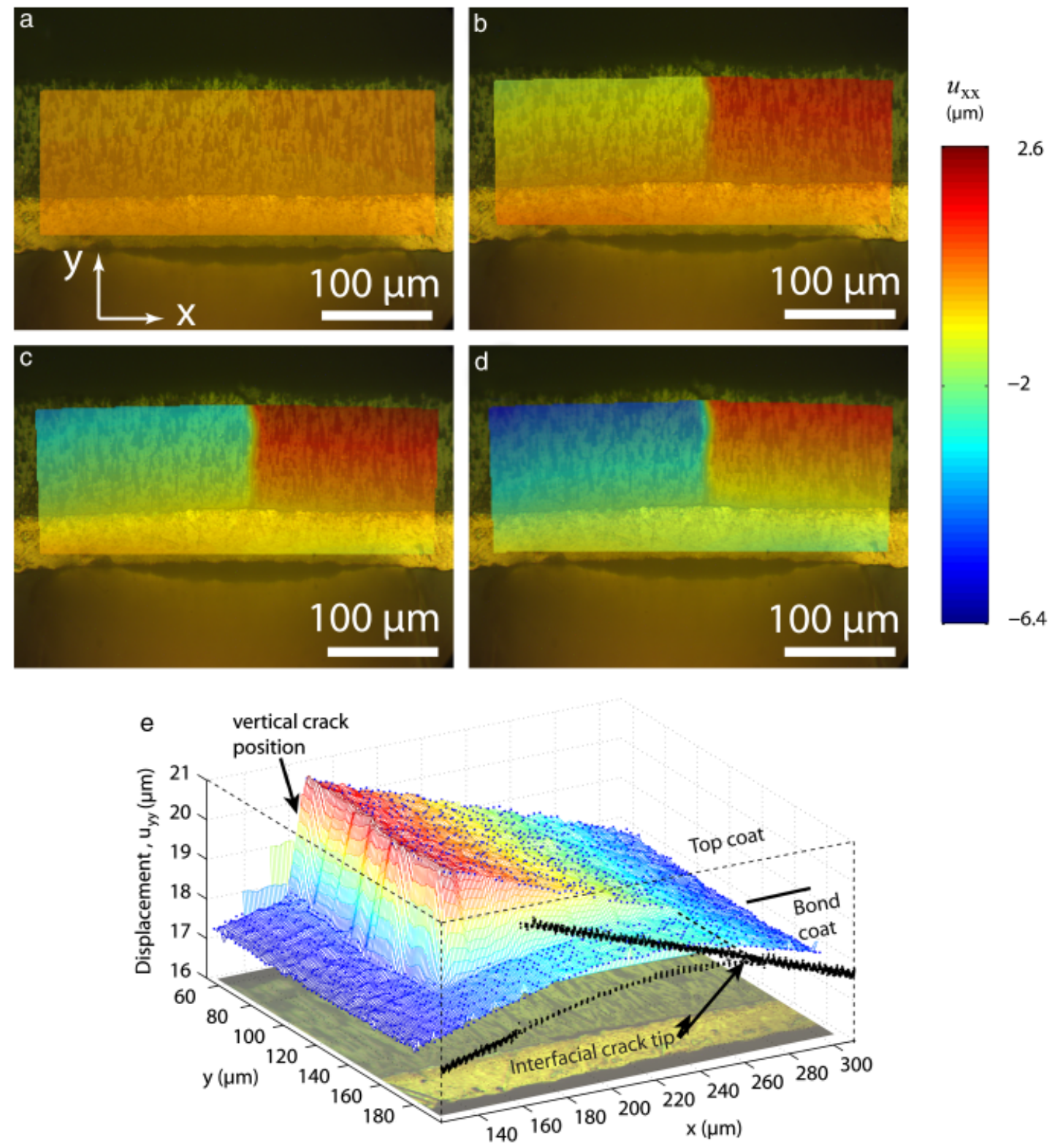

Fig. 3. The resulting full-field displacement $u_{x x}$ in the unloaded (a) as well as cracked and loaded (b)-(d) is shown in the upper images. The $3 \mathrm{D}$ deformation plot (e) shows how the crack tip can be measured from the full-field displacement data.

TBC is assumed aniostropic in material property: the in-plane modulus, $E_{\Pi}$, is considered to be $30 \mathrm{GPa}$ according to an elasticity study of the same material, ${ }^{8}$ and the out-of-plane modulus, $E_{\perp}$, is assumed to be $150 \mathrm{GPa}$ (compared with $E_{\perp} \approx 200 \mathrm{GPa}$ for dense YSZ). Poisson's ratio of TBC is considered to be 0.2 . The bond coat is considered to be elastic/plastic with power law hardening having the stress/strain curve obtained in Kim et al. ${ }^{9}$ (Young's modulus $E=155 \mathrm{GPa}$, Poisson's ratio $v=0.25$, yield strength $\sigma_{\mathrm{Y}}=750 \mathrm{MPa}$, and strain hardening exponent $n=0.2$ ).

\section{(1) Basic Features}

ABAQUS calculations based on the appropriate specimen dimensions and material properties allows the energy release rate and mode mixity to be determined as a function of the crack length. The energy release rate, i.e. the $J$-integral, is calculated in ABAQUS for the interface crack as the loading increases. The results are presented in the normalized coordinates suggested by beam theory, ${ }^{5} G_{\mathrm{p}} E_{b c} h_{b c}^{3} /\left(P_{\mathrm{c}}\right)^{2}$ as a function of $a / L$ (Fig. 6). Note that, at fixed load, the energy release rate decreases as the crack extends, reaffirming the stable nature of this test configuration. The stress fields and mode mixity of an interfacial crack has been shown to have an oscillatory character in a bimaterial specimen. These oscillations were observed in the FE simulations and the associated mode mixity varied with distance from the crack tip. ${ }^{1}$ At a distance of $1 \mu \mathrm{m}$, which corresponds with the plastic zone size in the FE simulation, the mode mixity was calculated to be $20^{\circ}$. The mode mixity increased to approximately $40^{\circ}$ at a distance of $8-10 \mu \mathrm{m}$. This larger value approaches the mode mixity that is traditionally reported for four-point flexure measurements, but is still slightly smaller ${ }^{1,2}$ for the other methods that have been used to measure TBC interfacial toughness. ${ }^{9-12}$

\section{(2) Experimental Findings}

Specific values of fracture resistance, $\Gamma_{R}$, have been calculated for each of the critical crack growth events reported in the previous section. The calculated $J$-integral at the critical load is considered as the interface toughness. These fracture resistance values are included in Table I, and inspection of the data indicates that the measured toughness values fall into a rather broad range $\left(25-95 \mathrm{~J} / \mathrm{m}^{2}\right)$, mimicking perhaps the tortuosity of the $\mathrm{TGO} /$ bond coat interface. It is worth noting that the measured 

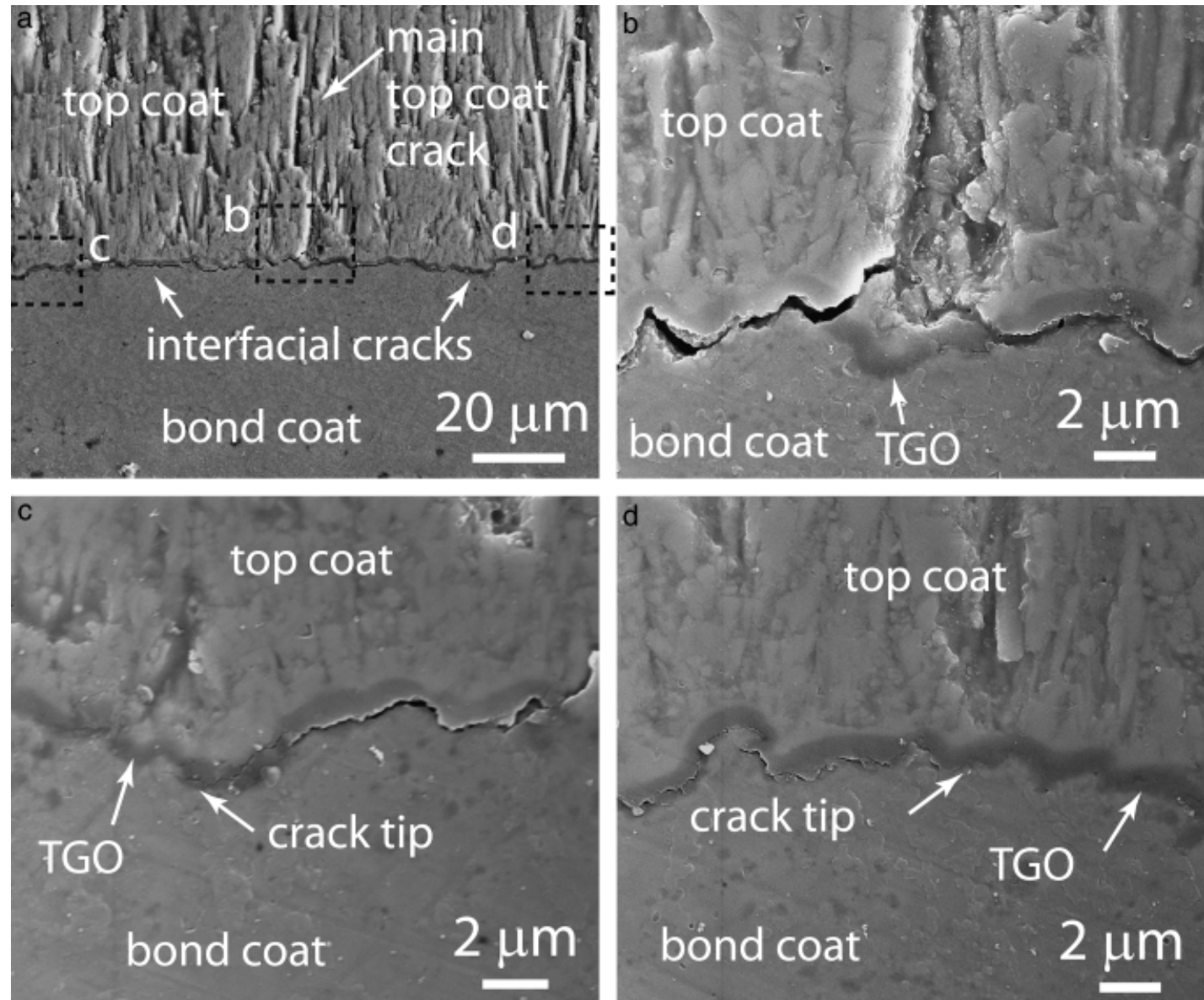

Fig. 4. Scanning electron micrographs of the region near the center of a test specimen reveal both, precrack (a) and delamination (b), (c) and (d) at the interface between the TGO and the bond coat.

fracture resistance appears to rise with crack length in both data sets. This rise cannot be attributed to changes in mode mixity and may be indicative of a rising resistance with crack extension. This could be explained if the crack may arrest at selectively tougher features on the tortuous interface. Alternatively, the role of permanent plastic deformation in the bond coat has not been completely ruled out. It is also possible that the TBC was damaged when the sample was initially loaded. No evidence of damage was noted, but it is possible that undetected damage resulted in the lower value of interfacial toughness for the first event in Table I. Given this uncertainty, we prefer not to ascribe any significance to the variation in toughness with mode mixity and to represent our measurement with the mean of all measurements. The mean values of the toughness for each individual

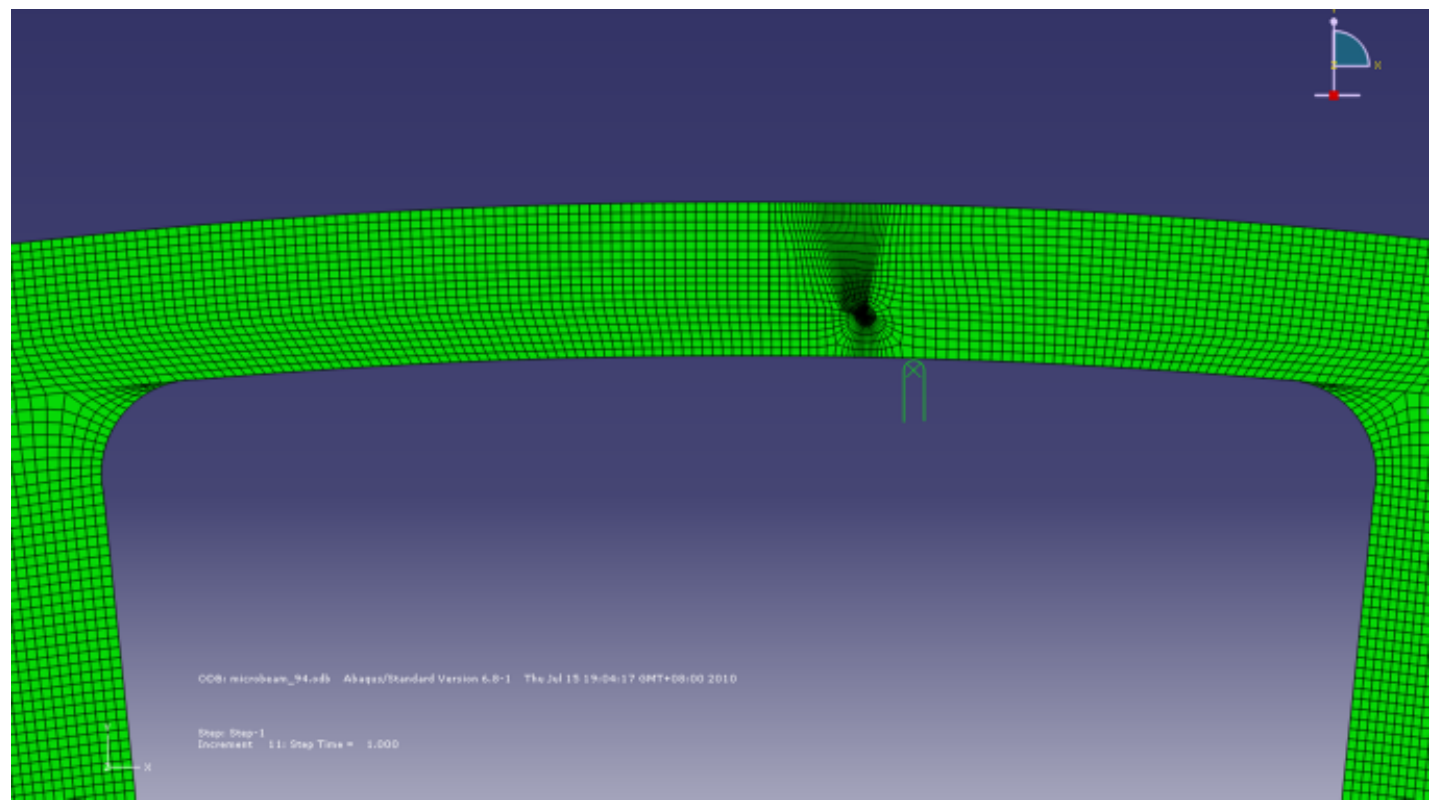

Fig. 5. The finite-element model and the mesh, with the refined mesh close at the crack tip. 


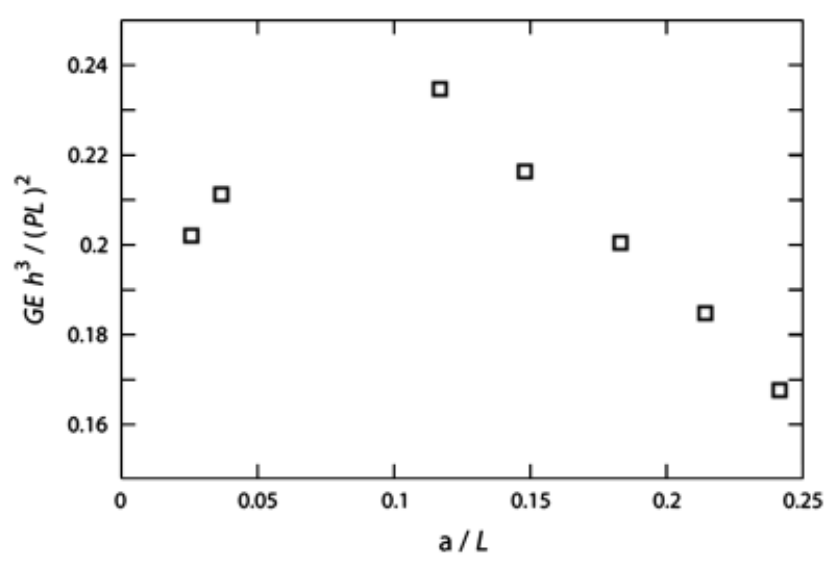

Fig. 6. Normalized plots of FE results, e.g. normalized $J$ and mode mixity as a function of $a / L$. (a) The nondimensional energy release rate as a function of relative interface crack length for the asymmetric singlepoint test, see event table for geometric details. The phase angle shows a constant value until the crack tip is closing in on the off center load tip position (b).

beam were measured to be nearly identical and the overall mean value for all toughness measurements was determined to be $57.3 \pm 21.5 \mathrm{~J} / \mathrm{m}^{2}$.

\section{(3) Influence of Residual Stress}

One important but often overlooked caveat in the measurement of interfacial toughness is the fact that potential residual stresses in the TBC will influence the energy release rate, as well as the mode mixity. These residual stresses could result from fabrication processes in which the sample is subjected to different temperatures. Because the materials involved have different thermal expansion coefficients, significant thermal stresses arise in the individual layers with different signs. Furthermore, in service TBC layers are also exposed to stresses from growth, sintering and changes in chemical composition leading to a phase evolution. Theses stresses are additive to those from thermo-mechanical cycling and can result, together with creep processes, in a difficult to predict stress distribution. One advantage of the test methodology developed in this study is the fact that the residual stresses in the bilayer beam are relaxed during specimen preparation, during $\mu$-EDM removal of the underlying substrate. This salient feature was illustrated in Eberl et al., ${ }^{8}$ where one edge of a double-end-clamped bilayer beam was cut through and the relative displacement between the shoulder and the free-end of the beam was undetectable. This finding holds practical significance in that it obviates the need to correct the measured toughness data to account for the effects of residual stress in the test specimens.

\section{Discussion}

A new test methodology that can be used to measure in situ the delamination toughness of coatings or films attached to both test specimens and components has been developed. The use of $\mu$-EDM to remove a section of the substrate and create a coating/substrate bilayer beam provides a robust specimen that can be easily and stably loaded. Digital image correlation provides for direct high-fidelity measurement of crack length, which can be used to measure the critical load for crack extension. FE analysis can and has been used to estimate the associated energy release rates and mode mixity from these measurements.

The efficacy of this new methodology is demonstrated through in situ measurement of the toughness of the interface between an as-deposited TBC and its underlying NiCoCrAlY bond coat on a standard burner rig bar. The toughness values measured in this study $\left(57.3 \pm 21.5 \mathrm{~J} / \mathrm{m}^{2}\right)$ are compared with literature values of toughness that were obtained using other

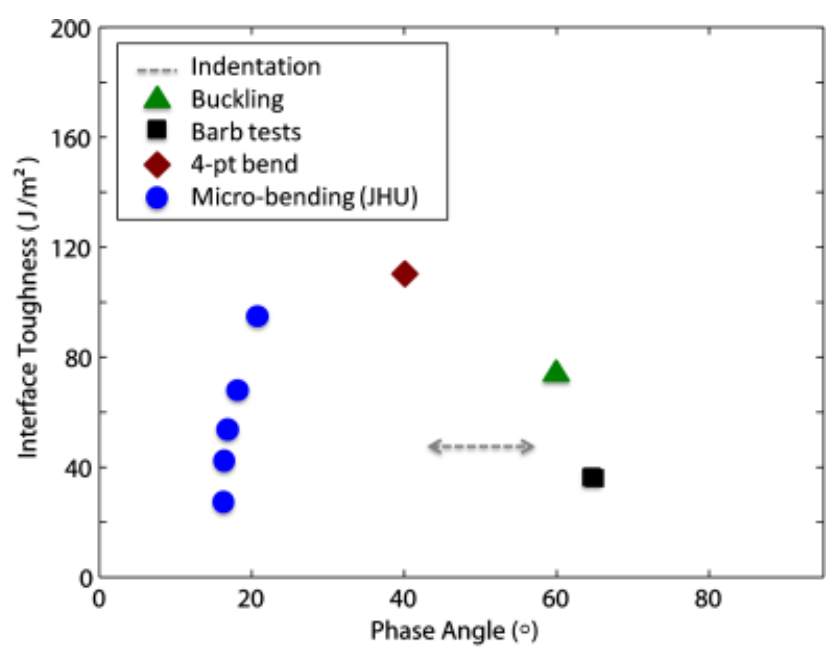

Fig. 7. Experimentally determined values of EBPVD TBC interfacial toughness plotted as a function of mode mixity. The average value of toughness measured in the current study is in reasonable agreement with data published previously. The scatter in this data, which were obtained by a variety of techniques with differing assumptions of modulus and residual stress, masks any possible dependence of toughness on mode mixity.

methodologies (Fig. 7). Vasinonta and Beuth ${ }^{13}$ used the size of the delamination that occurs upon indentation to estimate the interfacial toughness of as-deposited EB-PVD TBC samples to be $49 \mathrm{~J} / \mathrm{m}^{2}$, assuming a residual stress of $50 \mathrm{MPa}$ and anisotropic TBC modulus of $44 \mathrm{GPa}$. Faulhaber et al. ${ }^{11}$ analyzed the shape of the coating in buckled regions adjacent to spalled areas of burner rig bars identical to those examined in the current study. Establishing the mechanics of ridge-cracked buckle delaminations for multilayers on curved substrates allowed them to predict delamination toughness; assuming a TBC modulus of $50 \mathrm{GPa}$ they predicted the interfacial toughness to be $75 \mathrm{~J} / \mathrm{m}^{2}$ for a mode mixity of $60^{\circ}$. Guo and colleagues implemented a barb pullout test for EB-PVD TBC and initially reported an interfacial toughness of $45 \mathrm{~J} / \mathrm{m}^{2}$ and a phase angle of $90^{\circ}$, but Liu and colleagues reevaluated these tests by taking into account residual stresses and reported an interfacial toughness of $36 \mathrm{~J} / \mathrm{m}^{2}$ and a phase angle of $65^{\circ}$ for Young's modulus of $44 \mathrm{GPa}$. Thery et al. ${ }^{14}$ used a double cantilever experiment to measure fracture toughness ${ }^{3}$ of EB-PVD and reported a value of $110 \mathrm{~J} / \mathrm{m}^{2}$ but did not mention the modulus or residual stresses. The phase angle for such a test should be roughly $40^{\circ} .{ }^{15}$ Similar experiments have been carried out by Bahr et al. ${ }^{16}$ who reported a value of $>81 \mathrm{~J} / \mathrm{m}^{2}$ at which point their stiffening layer detached.

One additional benefit of the new test methodology is that it provides a measure of the interfacial toughness for values of mode mixity that are different than measured previously. The values of mode mixity predicted by the FE analysis of the microbend experiments fall in the range of $20^{\circ}-40^{\circ}$, which is slightly closer to mode I than has be reported for other test methods. Hutchinson and Hutchinson ${ }^{17}$ have shown that the delamination of a TBC occurs as a result of mode II loading. In this regard the ability to measure the mode II interfacial toughness would be most straightforward; nevertheless, the ability to measure interfacial toughness over a broad range of mode mixity is also desirable.

Two additional benefits of the new specimen geometry and test methodology include: (i) the relaxation of the residual stresses in the test specimen and (ii) the natural extension of this technique to the characterization of engine hardware. With regard to the former, the realization that the residual stresses in the bilayer beam are relaxed when the underlying superalloy section is removed by $\mu$-EDM simplifies the analysis of the test. Although often overlooked residual stress can have a very large 
influence on measured values of interfacial toughness, and in is helpful to have the importance of residual stresses in reduced during specimen preparation. The results obtained for the study outlined in this article were for as-deposited TBC coatings, but the use of this technique to measure the influence of thermal cycling, external deposits (e.g., CMAS), TBC sintering and phase evolution are envisioned. Of particular interest is the ability to use this in situ technique to characterize engine hardware. Slicing, $\mu$-EDMing, and characterizing commercial components provide an opportunity to measure location specific properties as well as the influence of the turbine environment.

\section{Acknowledgments}

The authors would like to thank J. Hutchinson for creative and enlightening discussions that allowed this technique to come to fruition. We also thank M. Maloney and D. Litton of Pratt and Whitney (USA) for providing the specimens, S. Faulhaber for preparing the disk specimens, J. Mraz (Smaltec) for fabricating the microbeam specimens, and W. Sharpe, Jr. and J. Sharon for technical support. C. E. would like to acknowledge financial support from the German Science Foundation (SFB499/3-2007 N01).

\section{References}

'J. Hutchinson and Z. Suo, "Mixed Mode Cracking in Layered Materials," $A d v$. Appl. Mech., 29, S63-191 (1992).

${ }^{2}$ A. A. Volinsky, N. R. Moody, and W. W. Gerberich, "Interfacial Toughness Measurements for Thin Films on Substrates," Acta Mater., 50, S441-66 (2002).

${ }^{3}$ P. G. Charalambides, J. Lund, A. G. Evans, and R. M. McMeeking, "A Test Specimen for Determining the Fracture Resistance of Bimaterial Interfaces," J. Appl. Mech., 56, 77-83 (1989).

${ }^{4}$ P. G. Charalambides, H. C. Cao, J. Lund, and A. G. Evans, "Development of a test method for measuring the mixed mode fracture resistance of bimaterial interfaces," Mech. Mater., 8, S269-83 (1990).
${ }^{5}$ M. Y. He, J. W. Hutchinson, and A. G. Evans, "A Stretch/Bend Method for In Situ Measurement of the Delamination Toughness of Coatings and Films Attached to Substrates," J. Appl. Mech., 78 [1] S011009, 5pp 2010.

${ }^{6} \mathrm{~K}$. Hemker, B. Mendis, and C. Eberl, "Characterizing the Microstructure and Mechanical Behavior of a Two-Phase NiCoCrAlY bond coat for thermal barrier systems," Mater. Sci. Eng. A, 483-484, S727-30 (2008).

${ }^{7}$ C. Eberl, D. Gianola, and K. Hemker, "Mechanical Characterization of Coatings Using Microbeam Bending and Digital Image Correlation Techniques," Exp. Mech., 50, S85-97 (2010).

${ }^{8}$ C. Eberl, D. S. Gianola, X. Wang, M. Y. He, A. G. Evans, and K. J. Hemker, "A Method for In Situ Measurement of the Elastic Behavior of a Columnar Thermal Barrier Coating," Acta Mater., 59 [9] 3612-20 (2011).

${ }^{9}$ S. Kim, Y. Liu, and Y. Kagawa, "Evaluation of Interfacial Mechanical Properties under Shear Loading in EB-PVD TBCs by the Pushout Method," Acta Mater., 55, S3771-81 (2007).

${ }^{10}$ Y. Liu, Y. Kagawa, and A. Evans, "Analysis of a "Barb Test" for Measuring the Mixed-Mode Delamination Toughness of Coatings," Acta Mater., 56, S43-9 (2008).

${ }^{11}$ S. Faulhaber, C. Mercer, M. Moon, J. Hutchinson, and A. Evans, "Buckling Delamination in Compressed Multilayers on Curved Substrates with Accompanying Ridge Cracks," J. Mech. Phys. Solids, 54, S1004-28 (2006).

${ }^{12}$ S. Guo, Y. Tanaka, and und Y. Kagawa, "Effect of Interface Roughness and Coating Thickness on Interfacial Shear Mechanical Properties of EB-PVD YttriaPartially Stabilized Zirconia Thermal Barrier Coating Systems," J. Eur. Ceram. Soc., 27, S3425-31 (2007).

${ }^{13}$ J. L. Vasinonta and A. Beuth, "Measurement of Interfacial Toughness in Thermal Barrier Coating Systems by Indentation," Eng. Fracture Mech., 68, S84360 (2001).

${ }^{14}$ P. Thery, M. Poulain, M. Dupeux, and M. Braccini, "Adhesion Energy of a YPSZ EB-PVD Layer in Two Thermal Barrier Coating Systems," Surf. Coat. Technol., 202, S648-52 (2007).

${ }^{15}$ R. H. Dauskardt, M. Lane, Q. Ma, and N. Krishna, "Adhesion and Debonding of Multi-Layer Thin Film Structures,” Eng. Fracture Mech., 61, S141-62 (1998).

${ }^{16}$ H. Bahr, H. Balke, T. Fett, I. Hofinger, G. Kirchhoff, D. Munz, A. Neubrand, A. S. Semenov, H.-J. Weiss, and Y. Y. Yang, "Cracks in Functionally Graded Materials," Mater. Sci. Eng. A, 362, S2-16 (2003).

${ }^{17}$ R. G. Hutchinson and J. W. Hutchinson, "Lifetime Assessment for Thermal Barrier Coatings: Tests for Measuring Mixed Mode Delamination Toughness,' J. Am. Ceram. Soc., 2011, doi:10.1111/j.1551-2916.2011.04499.x. 